\title{
Comparison of Single-Step Recovery Anneal Processing and a Single-Step Recrystallization Processing on the Generation of Special Boundaries in Commercially Pure Nickel
}

\author{
Qiangyong Li • N. L. Richards
}

Received: 2 August 2012/Revised: 23 October 2012/Accepted: 19 November 2012/Published online: 12 December 2012

(C) Springer Science+Business Media New York and ASM International 2012

\begin{abstract}
The strain recovery route to generate a significant increase in special boundaries nominally involves a strain level of less than about $10 \%$, plus a recovery anneal at a temperature-time combination sufficient to cause grain boundary migration, but insufficient cold work/time/temperature combinations to cause recrystallization of the material being processed. In the present communication using a simple material to avoid complications from industrial alloys, commercially pure (C.P.) $\mathrm{Ni}$ was processed using a single low strain value of $6 \%$ and a temperature of $800-1000{ }^{\circ} \mathrm{C}$ for time up to $38 \mathrm{~min}$. The fraction of special grain boundaries, $F_{\mathrm{sp}}$, based on a $\Sigma 3-29$ as evaluated by Orientation Image Microscopy was then compared with the as-received C.P. Ni and $\mathrm{Ni}$ strained $25 \%$ and annealed at $800{ }^{\circ} \mathrm{C}$ to give a recrystallized structure. For the single low strain route, a $6 \%$ deformation at a temperature of $800{ }^{\circ} \mathrm{C}$ and a time of 8-10 min resulted in an $F_{\mathrm{sp}}$ value of close to $80 \%$, as well as a grain size of about $25-35 \mu \mathrm{m}$ compared to the as-received value of about $25 \mu \mathrm{m}$. Thus, while grain boundary migration occurred under these processing conditions, the increase in grain size was not excessive and would not result in a large reduction in tensile properties. The single $25 \%$ rolled and $800{ }^{\circ} \mathrm{C}$ recrystallized test samples however resulted in no major increase in $F_{\text {sp }}$ relative to the as-received material, both being in the range 35-40\% over a range of times. Therefore, compared to the optimized $F_{\text {sp }}$ low strain recovery values of close to $80 \%$, the fully recrystallized $F_{\mathrm{sp}}$ values were approximately half. It should be noted however that multiple cycles of higher strain values rather than a single strain value, coupled with a
\end{abstract}

Q. Li · N. L. Richards $(\bowtie)$

Department of Mechanical \& Manufacturing Engineering, University of Manitoba, Winnipeg, MB R3T 5V6, Canada e-mail: nrichar@cc.umanitoba.ca recrystallized anneal, have been shown in the literature to result in increase in the $F_{\text {sp }}$ levels.

Keywords Grain boundary engineering - Thermomechanical processing - Orientation image microscopy . Nickel

\section{Introduction}

Grain boundary engineering (GBE) refers to the process of controlling a material's properties by altering the grain boundary character, usually by promoting the formation of special grain boundaries. GBE is an important design and processing technique in that it has been shown to significantly affect many important material properties such as corrosion, fracture behavior, creep, and weldability [1]. The GBE process can be divided into four main categories: onestep strain recovery, iterative strain recovery, one-step strain recrystallization, or iterative strain recrystallization. Different processes are suitable to different materials as, even for the same method, different parameters such as the percentage strain, recovery, recrystallization temperature, annealing time, or number of cycles may also cause a big difference of coincident site lattice (CSL) fractions and distributions. Here, the CSL is defined as the reciprocal density of the fraction of coincident lattice sites. The following is a brief introduction to some of the main research in the literature.

\section{One-Step Strain Recovery Process}

This process was shown to be effective in improving the fraction of CSL boundaries in several studies [2-5]. Under these processing conditions, one needs to ensure that grain 
boundary migration occurs, but not recrystallization. Thus, a combination of certain values of strain, usually $<10 \%$, combined with a temperature insufficiently high to cause major recrystallization, but high enough to cause grain boundary migration is needed. This usually results in a coarser grain size than the initial value, though one strives to optimize the generation of $\Sigma 3^{n}$ type boundaries at the expense of random boundaries. Thus, for the strain annealing process, grain growth is necessary, but should be controlled as not to be excessive, with a concomitant reduction in overall tensile properties.

In the work of Lim and Raj [6], 270-grade pure $\mathrm{Ni}$ was strained 5\% at room temperature and then annealed at $1573 \mathrm{~K}$ for $10 \mathrm{~min}$. About $40 \%$ of all boundaries were twin boundaries, and about $51 \%$ of these were coherent twin boundaries. More than half of CSL boundaries were boundaries with $\Sigma \leq 81$, and $87 \%$ of them were $\Sigma 3,9$, and 27. Unfortunately these authors did not state the baseline CSL values.

Lee and Richards [2] used the low strain method to improve grain boundary characteristic of commercial pure $\mathrm{Ni}(99.5 \%)$. After the material was strained $6 \%$ and annealed at $900{ }^{\circ} \mathrm{C}$ for $10 \mathrm{~min}$, the fraction of CSL was increased from 36.5 to $74.7 \%$, and a large fraction of the increased CSL boundaries consisted of annealing twin boundaries.

\section{Iterative Strain Recovery Process}

Iterative strain recovery is another way to improve CSL boundary fractions [7]. Was et al. [7] processed alloy 600 (Ni-16Cr-9Fe) by multiple cycle steps of low level deformation (2-5\%) followed by annealing at $890-940{ }^{\circ} \mathrm{C}$ from 1 to $10 \mathrm{~h}$. This processing increased the proportion of $\Sigma 3 \mathrm{~s}$ and $\Sigma 9$ s from 6 to $12 \%$ and 5 to $12 \%$, respectively, a marked improvement in properties over the single-step processing. The most successful low strain-anneal treatment of copper was found to be $6 \%$ compression followed by $14 \mathrm{~h}$ at $275{ }^{\circ} \mathrm{C}$ then $7 \mathrm{~h}$ at $375^{\circ} \mathrm{C}$, resulting in $67 \%$ of $\Sigma 3 \mathrm{~s}$ and $9 \%$ of $\Sigma 9 \mathrm{~s}$. $\mathrm{Li}$ et al. [8] studied the effect of multi-cycle processing on the grain boundary characteristics of 718 Superalloy. Sequentially processed samples were cold rolled to $2.5,5$, $7.5 \%$ strain, followed by annealing at $1020{ }^{\circ} \mathrm{C}$ for $10 \mathrm{~min}$. Their results suggested, after two cycles of processing, the $5 \%$ strained samples exhibited sharp increases in $F_{\mathrm{sp}}$ from as-received value of $26-65.5 \%$ after processing.

\section{One-Step Strain-Recrystallization Process}

A medium-to-high level of cold work followed by a very short high-temperature anneal can result in a small grain size (brought about by recrystallization) and result in a high fraction of twinning during the new grain formation and growth process. Romero and Murr [9] used a treatment consisting of $70 \%$ cold rolling followed by annealing at $1000{ }^{\circ} \mathrm{C}$ for $1 \mathrm{~min}$ to deliberately generate a high fraction of twins in 304 stainless steel. Sometimes, however, the results of the one-step deformation followed by a recrystallization treatment are better than a multi-step route, as illustrated by Randle [10] in copper using 50\% deformation plus $1000{ }^{\circ} \mathrm{C}$ for $1 \mathrm{~min}$. Higher proportions of twins were obtained, but the results regarding proportions of CSL boundaries were not reported.

\section{Iterative Strain-Recrystallization Process}

Multi-step recrystallization treatment using strain levels of 20-30\% and temperatures high enough to cause recrystallization was the first method used by Palumbo and co-workers to process alloys with high CSL boundary fraction, small grain size, and near-random texture (isotropic) characters. Palumbo [11] pointed out in his patent that austenitic stainless, iron-based or nickel-based FCC alloys, could be processed by $30 \%$ of strain, $2-10 \mathrm{~min}$ at $900-1050{ }^{\circ} \mathrm{C}$, and at least three deformation cycles, to produce a special grain boundary fraction of at least $60 \%$.

Lee [12] processed a commercial $\mathrm{Pb}$-based alloy used for automobile battery plates, for the purpose of enhancing the resistance against intergranular corrosion, cracking, and creep. Different percentages of strain from 30 to 90 and different strain annealing combinations were used, with ₹1-29 being considered to be CSL boundaries and boundaries with $\Sigma$ values above 29 being random. Heavy cold working (90\%), prior to two cycles of thermo-mechanical process with $30 \%$ strain and $270{ }^{\circ} \mathrm{C}$ recrystallization for 10 min improved the CSL proportion from about 20 to $91 \%$. Some $98 \%$ of these CSL boundaries were $\Sigma 3^{n}$ boundaries, which confirmed the prominent contribution of annealing twinning. In a similar way, strain-recrystallization treatments in copper of three cycles, comprising $30 \%$ strain and a 10 -min anneal at $375{ }^{\circ} \mathrm{C}$, increased the proportion of $\Sigma 3 \mathrm{~s}$ to $58 \%$ [12], also, five cycles of $20 \%$ strain and $5 \mathrm{~min}$ at $750{ }^{\circ} \mathrm{C}$ gave $45 \% \Sigma 3 \mathrm{~s}$ and $7 \% \Sigma 9 \mathrm{~s}$.

The present investigation continues previous research on the low strain recovery route using a fixed strain following previous publications [2, 3, 8] and annealed at various temperatures. The low strain route provides a simpler method than the multiple strain routes to increase the fraction of special boundaries, allowing the breakup of random boundary networks and improved material properties as outlined in section " 1. ."

\section{Experimental}

Commercially pure Ni 200 (99.5\% purity) sheet was used for this investigation. The $\mathrm{Ni}$ sheet was $3 \mathrm{~mm}$ thick and 
had been mill annealed at about $800{ }^{\circ} \mathrm{C}$. The nominal composition of the as-received material was $99.5 \mathrm{wt} . \% \mathrm{Ni}$ with minor amounts of $\mathrm{Fe}, \mathrm{Si}, \mathrm{Mn}, \mathrm{Cu}$, and $\mathrm{C}$ amounting to not $>0.5 \mathrm{wt} . \%$. The as-received material was cold rolled prior to heat treatment with the strain level being calculated as the percentage decrease in thickness after rolling. Test samples $75 \mathrm{~mm}$ long $\times 12.5 \mathrm{~mm}$ wide $\times 3 \mathrm{~mm}$ thick were first strained to $6 \%$ then annealed at 800,900 , and $1000{ }^{\circ} \mathrm{C}$ for different lengths of time to determine the effect of annealing time and temperature on the microstructure. The $F_{\mathrm{sp}}$ values ( $\left.\Sigma 3-29\right)$ were determined by orientation image microscopy with approximately 200-300 grain boundaries evaluated, per the recommendations of Alexandreanu and Was [13], to obtain an accuracy in measurement of about $\pm 10 \%$. Transmission electron microscopy (TEM) was carried out with electrolytically prepared samples taken from the centerline area of the sheet. The grain size was measured using the linear intercept from ASTM E112 with 600 intercepts counted, giving a relative accuracy of about $7 \%$. A strain recrystallized anneal test at $25 \%$ strain and $800{ }^{\circ} \mathrm{C}$ was also conducted to observe the effect of recrystallization on the formation and development of special boundaries relative to the strain recovery approach.

The $6 \%$ strained sample $F_{\mathrm{sp}}$ values were compared to the as-received material annealed at $800{ }^{\circ} \mathrm{C}$, with a onestrain recrystallized anneal test at $25 \%$ strain and an $800{ }^{\circ} \mathrm{C}$ anneal, to compare the effect of recrystallization on the formation and development of special boundaries.

\section{Results and Discussion}

Comparison of $1 \times 6 \%$ Strained Samples Annealed at Different Temperatures

A comparison of the " $F_{\mathrm{sp}}$ versus annealing time" relationships of as-received material and the $1 \times 6 \%$ strained samples annealed at 800,900 , and $1000{ }^{\circ} \mathrm{C}$ is shown in Fig. 1. It can be seen that the values of $F_{\mathrm{sp}}$ started to increase earlier at $1000{ }^{\circ} \mathrm{C}$ relative to that at 900 and
$800{ }^{\circ} \mathrm{C}$, with the resultant maximum $F_{\mathrm{sp}}$ values of around $80 \%$, which reflects the effect of temperature on the recovery anneal.

The " $F_{\mathrm{sp}}$ versus grain size" relationships of $1 \times 6 \%$ strained material annealed at 800,900 , and $1000{ }^{\circ} \mathrm{C}$ are shown in Fig. 2. The $F_{\mathrm{sp}}$ values increased with grain size from an as-received value of $35 \%$, becoming a maximum at around $80 \%$, this variation corresponding to the change of grain size from 25 to $45 \mu \mathrm{m}$; further grain growth did not increase the $F_{\mathrm{sp}}$ values. It can be seen that the $F_{\mathrm{sp}}$ values reached a maximum at smaller grain size at the lower temperature of $800{ }^{\circ} \mathrm{C}$, thus $800{ }^{\circ} \mathrm{C}$ is preferred to obtain samples with smaller grain size and high $F_{\text {sp }}$ values.

The driving force of boundary migration at a fixed strain level is determined by the heat treatment temperature and the length of time at temperature. A comparison of " $F_{\mathrm{sp}}$ versus temperature" relationships of $1 \times 6 \%$ strained material annealed at 1000,900 , and $800{ }^{\circ} \mathrm{C}$ is shown in Fig. 3. It can be seen that the annealing temperature did not change the maximum value of $F_{\mathrm{sp}}$, which is around $78 \%$, an indication that the formation of special boundaries is not simply temperature dependent.

A comparison of "twin density versus annealing time" relationships of $1 \times 6 \%$ strained samples heat treated at 800, 900, and $1000{ }^{\circ} \mathrm{C}$ is given in Fig. 4, with twin density being the number of twins per micron. The twin densities decreased and leveled off with the annealing time. Also, note from Fig. 1 that the value of $F_{\text {sp }}$ increased with the

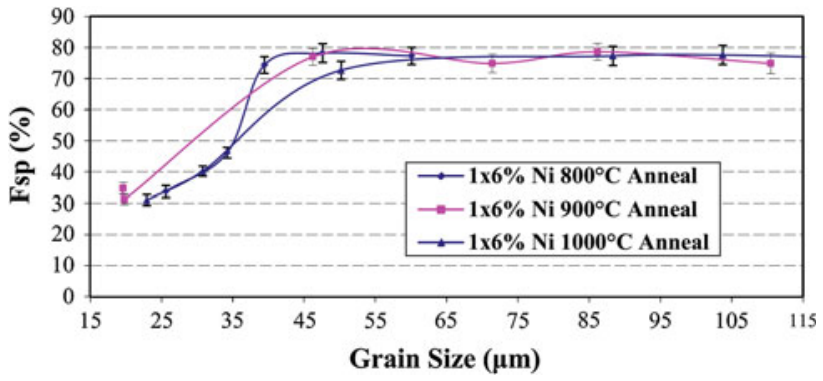

Fig. 2 "Grain size $-F_{\text {sp }}$ " relationships of $1 \times 6 \%$ strained material annealed at 800,900 , and $1000^{\circ} \mathrm{C}$ up to 38,25 , and $20 \mathrm{~min}$, respectively
Fig. 1 " $F_{\mathrm{sp}}$-annealing time" relationships of $1 \times 6 \%$ samples annealed at different temperatures

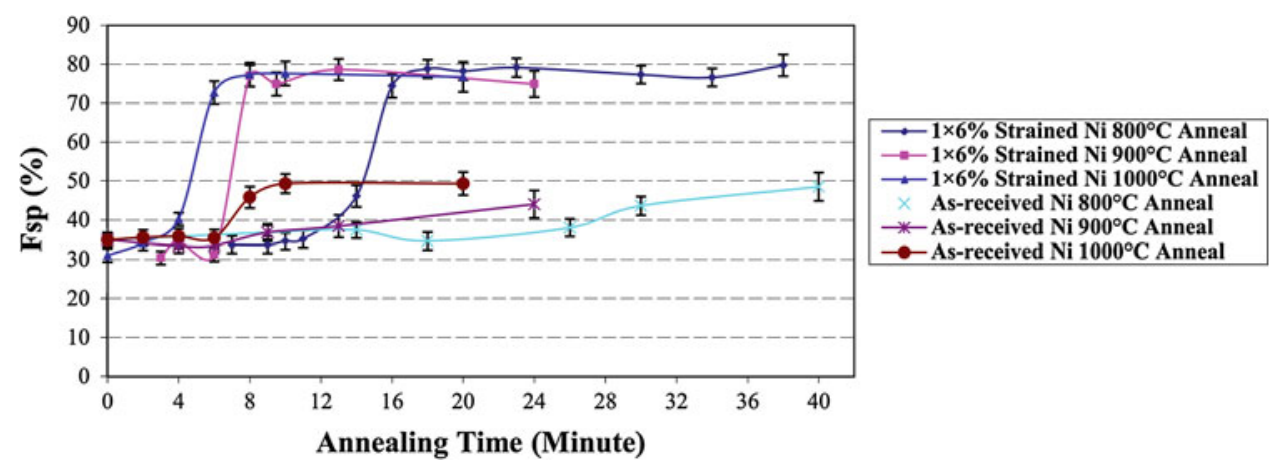




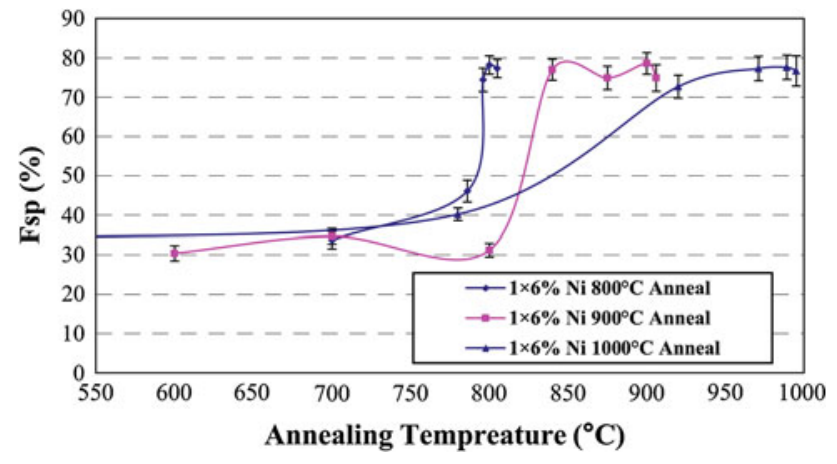

Fig. 3 " $F_{\mathrm{sp}}$-temperature" relationship of $1 \times 6 \%$ strained material annealed at 800,900 , and $1000{ }^{\circ} \mathrm{C}$ up to 38,25 , and $20 \mathrm{~min}$

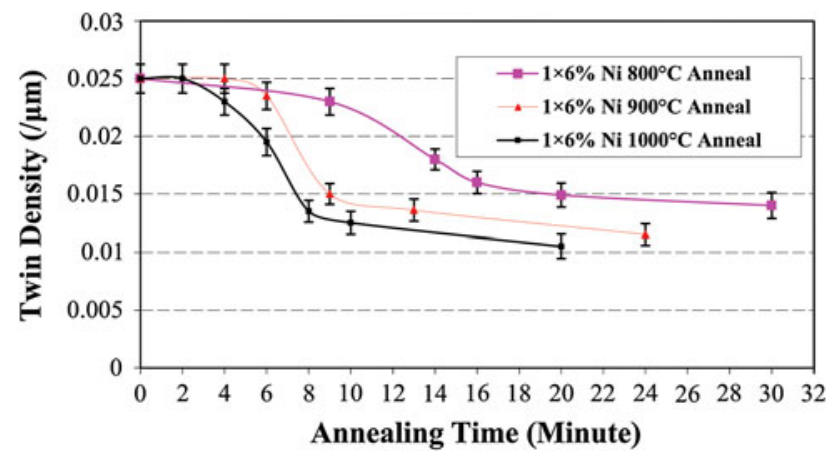

Fig. 4 "Twin density-annealing time" relationships of $1 \times 6 \%$ strained samples at different temperatures

annealing time, which suggests that the decreasing rate of high-angle boundary (HAB) was higher than that of special boundary in the process of annealing. The $F_{\mathrm{sp}}$ value of a material represents the relative amount of these two types of boundaries, which is determined by the equilibrium of production and annihilation. It can be observed in Fig. 1 that the $F_{\mathrm{sp}}$ values of $1 \times 6 \%$ strained samples prior to anneal are similar to the as-received material (about 35\%). However, the $F_{\text {sp }}$ values of strained samples (78\%) were almost 30\% higher compared to unstrained ones after annealing, indicating that the elimination rates of the HAB boundaries were sufficiently higher than that of special boundaries. It is reasonable to believe that the formation energy of HAB boundary (with more defects and crystal distortion) is higher than that of a special boundary causing less production of $\mathrm{HAB}$ boundaries in the process of grain growth.

In addition, the decrease of twin density started earlier at the higher temperature, a reflection of the thermal effect. Furthermore, the minimum value of twin density at $1000{ }^{\circ} \mathrm{C}$ was lower than that of 900 and $800{ }^{\circ} \mathrm{C}$.

A comparison of the "twin density versus grain size" relationships of $1 \times 6 \%$ strained samples annealed at 800 , 900 , and $1000{ }^{\circ} \mathrm{C}$ for different lengths of times is shown in Fig. 5. It can be seen that the twin density decreased

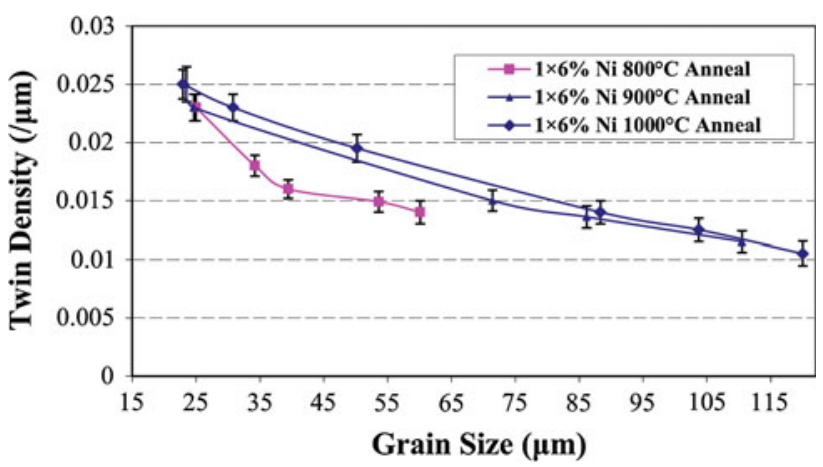

Fig. 5 Twin density-grain size relationship of $1 \times 6 \%$ strained samples heat treated at 800,900 , and $1000{ }^{\circ} \mathrm{C}$ for 38,25 , and $20 \mathrm{~min}$, respectively

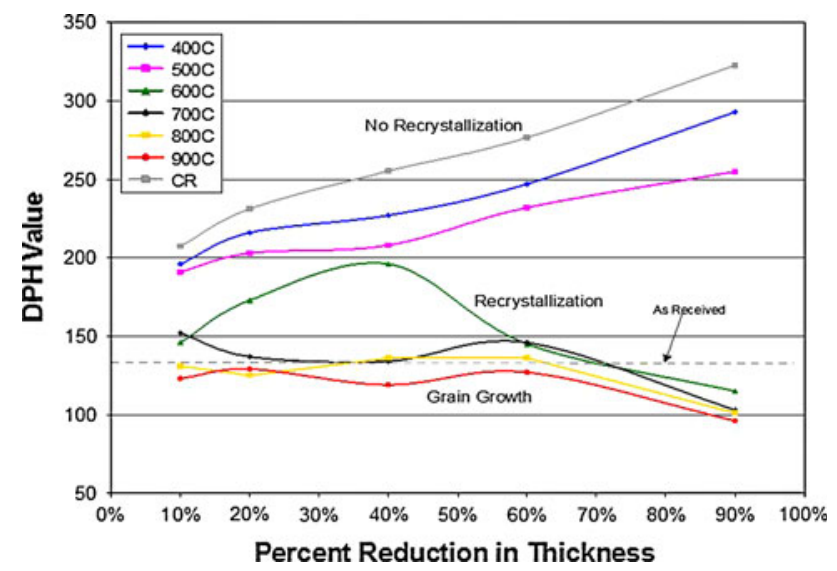

Fig. 6 Micro-hardness data of percent reduction in thickness versus annealing temperature [14]

with the increasing grain size in all cases, with each point plotted being for one strain-temperature-time combination.

One-Step of $25 \%$ Strain Annealed at $800{ }^{\circ} \mathrm{C}$ for Different Lengths of Time

Recrystallization may occur during the annealing of the heavily pre-deformed material, with recrystallization being used by some researchers to induce grain boundary modification. Figure 6 shows the effect of strain as a percentage reduction in thickness on rolling $3 \mathrm{~mm}$ strips, followed by annealing for $7 \mathrm{~min}$ from 400 to $900{ }^{\circ} \mathrm{C}$ in steps of $100{ }^{\circ} \mathrm{C}$ [14].

This data was used as a guide in the present experiment to evaluate a strain level when the C.P Ni would recrystallize at $800{ }^{\circ} \mathrm{C}$, for comparison with the $6 \%$ data strain annealed at $800{ }^{\circ} \mathrm{C}$. It can be seen from Fig. 6 that in the range of strain level tested (10-90\%), the $800{ }^{\circ} \mathrm{C}$ anneal for $7 \mathrm{~min}$ has a similar hardness to the as-received material 


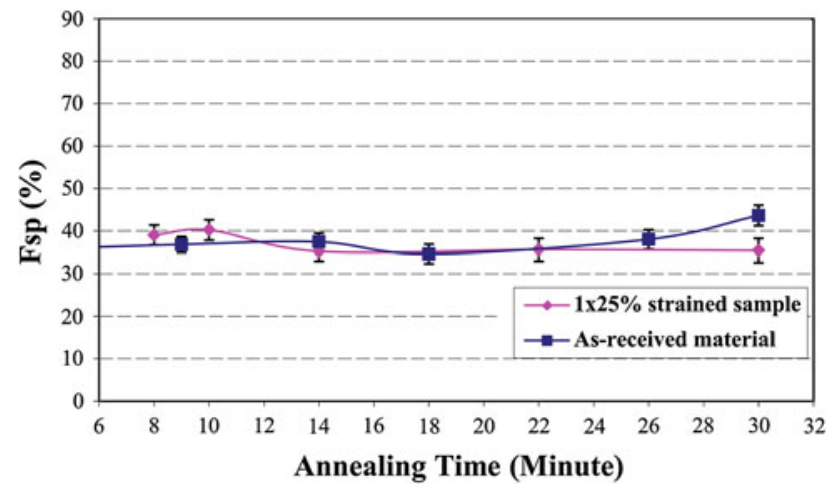

Fig. 7 " $F_{\text {sp }}$-annealing time" relationship of as-received material and the samples with $25 \%$ strain and annealed at $800{ }^{\circ} \mathrm{C}$ for different lengths of time

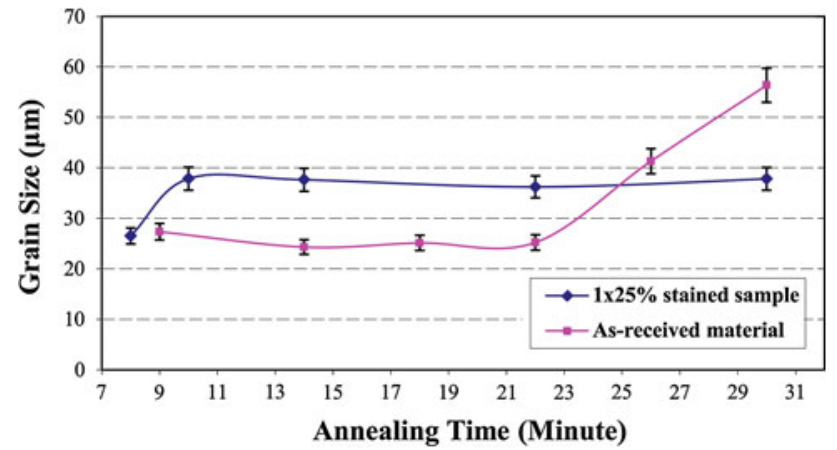

Fig. 8 Grain size of as-received and $1 \times 25 \%$ strained Ni annealed at $800{ }^{\circ} \mathrm{C}$ for different lengths of time

with a Vickers micro-hardness of about 135 (an indication of recrystallization).

The grain boundary configuration of the recrystallized material was studied by $25 \%$ pre-strained and $800{ }^{\circ} \mathrm{C}$ anneal. The comparison of " $F_{\mathrm{sp}}$ versus anneal time" relationship between unstrained and $1 \times 25 \%$ strained samples annealed at $800{ }^{\circ} \mathrm{C}$ is shown in Fig. 7. The $F_{\text {sp }}$ enhancement observed in the low strain experiments did not occur. The $F_{\mathrm{sp}}$ values of the $1 \times 25 \%$ strained material were unchanged with temperature, being distributed in the range of $35-40 \%$, similar to that of the unstrained material. (Fig. 8)

The grain size of the $1 \times 25 \%$ strained material increased with the time from original $26 \mu \mathrm{m}$ to a constant value of around $38 \mu \mathrm{m}$ after about $10 \mathrm{~min}$, which indicated that the driving force was not high enough for further grain boundary migration. As a comparison, grain growth started in the as-received material after about $22 \mathrm{~min}$, supporting the assumption that the original recrystallization annealing temperature of the as-received material was lower than $800{ }^{\circ} \mathrm{C}$

The " $F_{\text {sp }}$ versus grain size" relationship of the $1 \times 25 \%$ strained and unstrained material annealed at $800{ }^{\circ} \mathrm{C}$ is

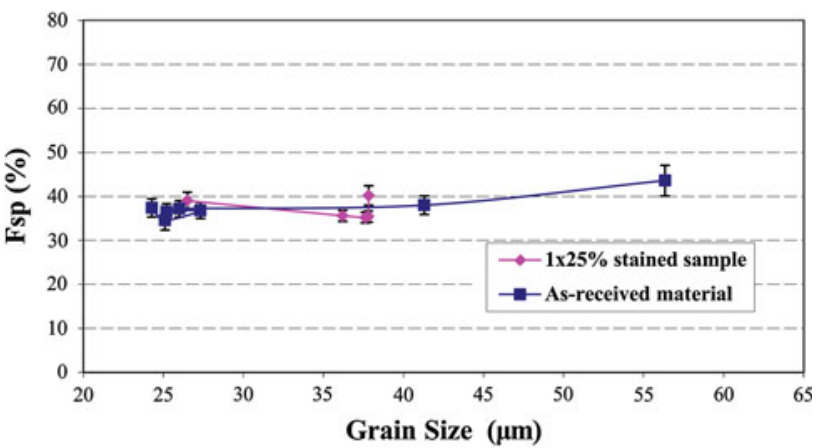

Fig. 9 " $F_{\text {sp }}$-grain size" relationship of samples with $25 \%$ strain and annealed at $800{ }^{\circ} \mathrm{C}$ for up to $30 \mathrm{~min}$

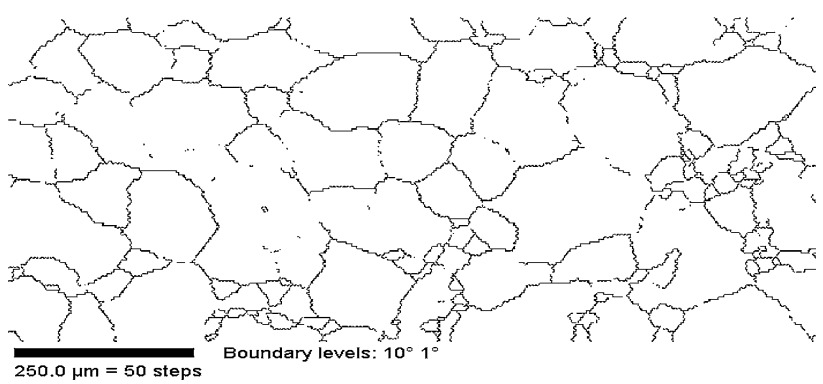

Fig. 10 Random boundary image of as-received Ni-200 annealed at $800{ }^{\circ} \mathrm{C}$ for $30 \mathrm{~min}$

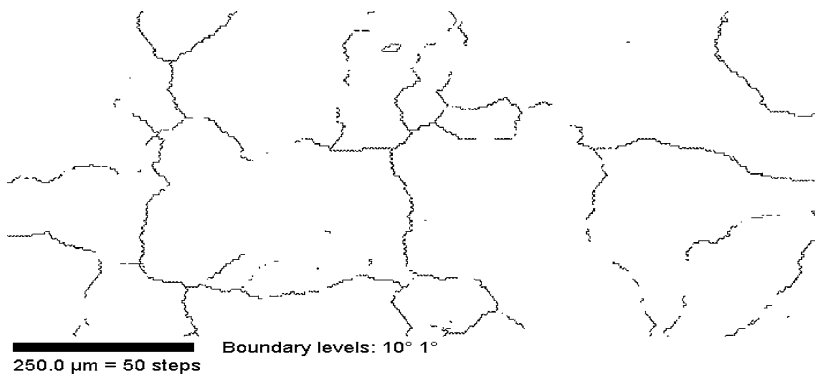

Fig. 11 Random boundary image of $1 \times 6 \%$ strained sample annealed at $800{ }^{\circ} \mathrm{C}$ for $30 \mathrm{~min}$

shown in Fig. 9. From the data, the $F_{\mathrm{sp}}$ values can be seen to be similar to that of the unstrained material. The $F_{\mathrm{sp}}$ values of the $1 \times 25 \%$ strained material do not increase significantly with grain size.

OIM images of the random boundary network for the asreceived, $1 \times 6 \%$ strain, and the $1 \times 25 \%$ strained materials all annealed at $800{ }^{\circ} \mathrm{C}$ for $30 \mathrm{~min}$ are shown in Figs. 10, 11, and 12, respectively.

Only the $1 \times 6 \%$ strain annealed at $800{ }^{\circ} \mathrm{C}$ shows apparent breakup of the random boundary network.

Thus, it can be concluded that in the present context that high strain levels and one cycle are not effective to the enhancement of $F_{\mathrm{sp}}$ values and improved boundary configurations. 


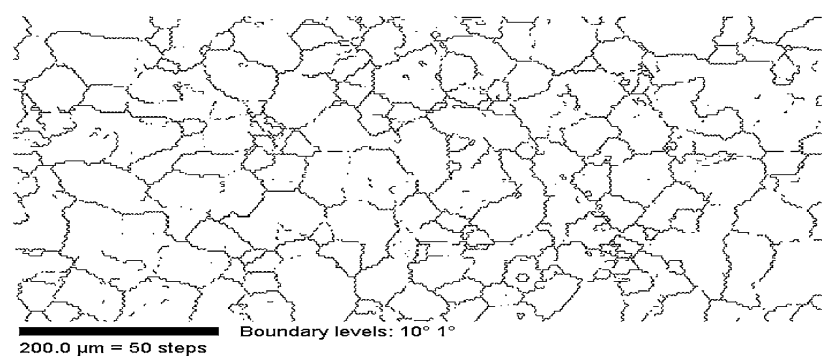

Fig. 12 Random boundary map of $1 \times 25 \%$ strained sample annealed at $800{ }^{\circ} \mathrm{C}$ for $30 \mathrm{~min}$

\section{Microstructural Effects of Low Strain}

Thompson et al. [15] have shown during tensile testing of polycrystalline metals, that non-homogenous deformation can occur adjacent to grain boundaries, leading to a concept of a harder "mantle" adjacent to the boundary and a softer "core" in the grain interior. Also, Meyers and Ashworth [16] showed that compatibility requirements at the grain boundary resulted in additional stresses $\tau_{i}$

$\tau_{i} / \sigma_{\text {ap }}=1.37$,

where $\sigma_{\mathrm{ap}}$ is the applied stress.

The shear stress at the interface is thus some three times that of the homogenously applied resolved shear stress, $\tau_{\mathrm{H}}=\sigma_{\mathrm{ap}} / 2$. They concluded that dislocation activity in the grain boundary area occurs before sources in the grain interiors activate. The result is that sources within the grain boundary activate first, leading to dislocations occurring in the vicinity of the boundary, before dislocation activity occurs within the body of the grain. Thus, on reaching the stress value needed to form dislocations, only deformation in the boundary vicinity occurs. Plastic flow in the grain boundary area then leads to reduction of the stress concentration and the generation of the geometrically necessary dislocations proposed by Ashby [17], resulting in micro-yielding. The result is a core-mantle dislocation arrangement with a concentration of geometrically necessary dislocations near the grain boundary and the statistically distributed dislocation in the bulk of the grain. Meyers and Ashworth developed the following equation for yield stress, $\sigma_{\mathrm{y}}$, based on a rule of mixtures equation:

$\sigma_{\mathrm{y}}=A_{\mathrm{B}} \sigma_{\mathrm{fB}}+A_{\mathrm{GB}} \sigma_{\mathrm{fGB}}$,

where $A_{\mathrm{B}}$ is the area of bulk grain, $A_{\mathrm{GB}}$ is the grain boundary area, $\sigma_{\mathrm{fB}}$ is the flow stress of bulk grain area, and $\sigma_{\mathrm{fGB}}$ is the flow stress of grain boundary area.

Further analysis using models approximating the grain size of a material and calculating the thickness of the grain boundary hardened layer $(t)$ resulted in the following expression [16]:

$$
\begin{aligned}
\sigma_{\mathrm{y}}= & \sigma_{\mathrm{fB}}+8 k\left(\sigma_{\mathrm{fGB}}-\sigma_{\mathrm{fB}}\right) D^{-1 / 2} \\
& -16 k^{2}\left(\sigma_{\mathrm{fGB}}-\sigma_{\mathrm{fB}}\right) D^{-1} .
\end{aligned}
$$

The term $k$ is related as $t=k D^{1 / 2}$, where $D$ is the grain size.

Thus Eq 3 predicts a $D^{-1 / 2}$ relationship at large grain sizes and as the grain size is reduced, the $D^{-1}$ term predominates. This was modelled by Meyers and Ashworth [16] using the data of Thompson et al. [15] for nickel, resulting in a good fit to the data. Thus, in line with Thompson et al., Meyers and Ashworth also concurred that dislocation emission would likely occur at grain boundary ledges, before initiation in the grain interior. Furthermore, the grain boundary emission of extrinsic grain boundary dislocations (EGBDs) would reduce the stress concentration for the generation of geometrically necessary dislocations, which accommodate these stresses. The grain boundary flow stress is therefore greater than the bulk value, which is the basis of the mantle-and-core proposal. On further straining, the elastic strained region becomes plastic and conventional macroscopic strain occurs. In this case, dislocations in the mantle and core will behave in a similar fashion, and the flow stress in both the grain boundary and the core will become similar.

Previous research by our group has shown that 2.5-6\% strain gives the highest $F_{\mathrm{sp}}$ values [2, 3]. It is therefore proposed that strain levels of $\sim 2.5-6 \%$ produce the mantle-core effect, whereas greater strains such as 9 and $12 \%$ result in the flow stress of the core and mantle being approximately the same; i.e., dislocations have spread throughout the grains. Thus, at low strains, recrystallization does not occur, whereas at higher strain levels, depending on temperature, recrystallization may occur and new highangle grain boundaries form, destroying the existing microstructure, which is conducive to the formation of special boundaries. Sangal and Tangri [18] have shown that, during annealing the non-equilibrium grain boundaries can be transferred to an equilibrium state via grain boundary migration, and the migration is related to dislocated annihilation in the area of the grain boundary. In the first stage, EGBD cores spread out into a number of partial dislocations, followed by a climb of these dislocations along the grain boundary plane toward the triple points. The annihilation of the EGBDs is a function of temperature and time and is quantitatively developed in [18].

\section{Transmission Electron Microscopy}

TEM observations of the $1 \times 6 \%$ strained material annealed at $800{ }^{\circ} \mathrm{C}$ for different lengths of time are shown in Fig. 13. Figure 13(a) is the micrograph of the as-received material, with no dislocation tangles or cells distributed near boundaries, indicating a recrystallized structure. A 

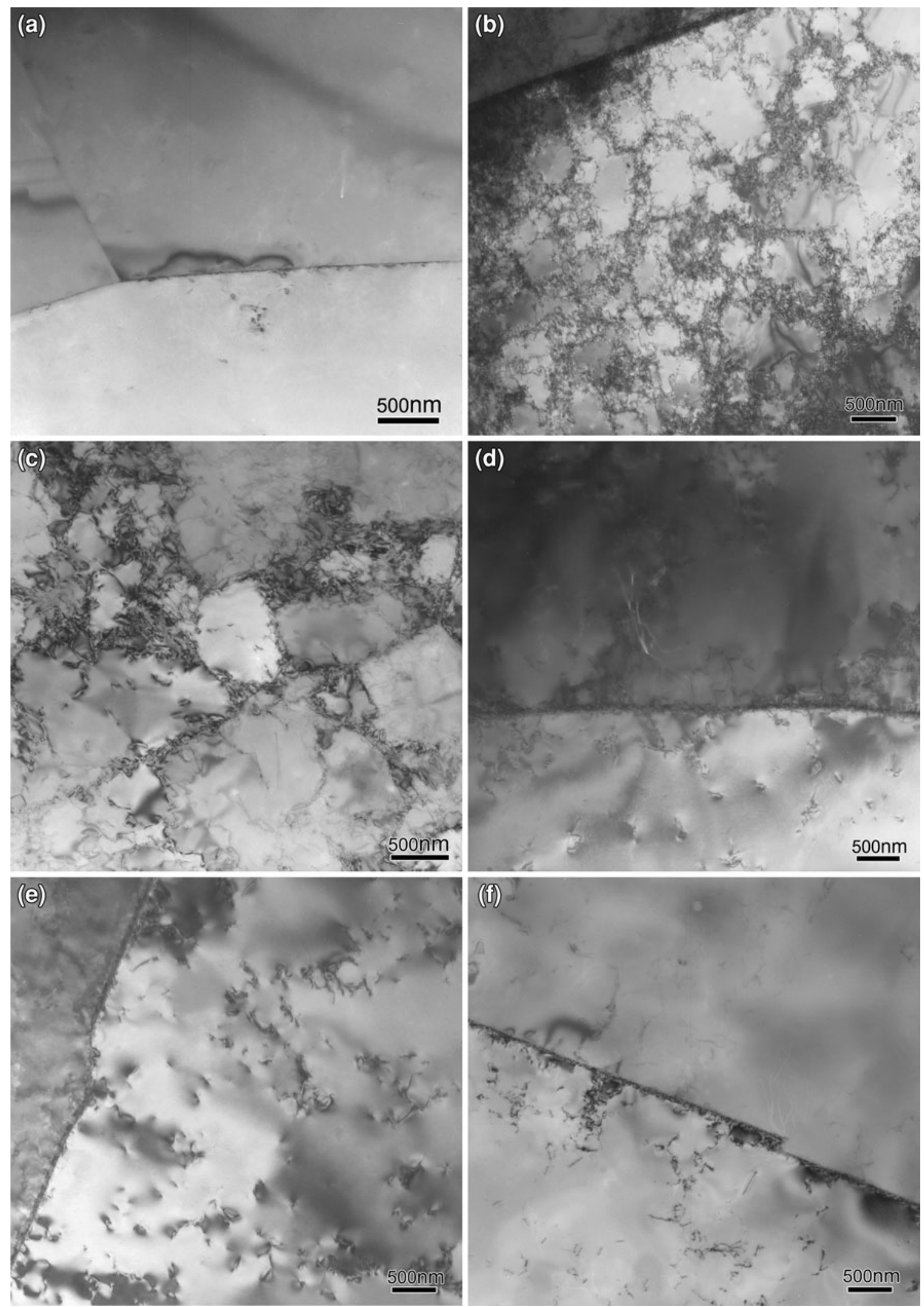

Fig. 13 TEM observation of $1 \times 6 \%$ strained samples annealed at $800{ }^{\circ} \mathrm{C}$ for different lengths of time: (a) as-received, (b) $1 \times 6 \%$ strained $\mathrm{Ni}-200$, (c) $1 \times 6 \% 800{ }^{\circ} \mathrm{C} 9 \mathrm{~min},(\mathrm{~d}) 1 \times 6 \% 800{ }^{\circ} \mathrm{C} 14 \mathrm{~min}$, (e) $1 \times 6 \% 800{ }^{\circ} \mathrm{C} 16 \mathrm{~min}$, and (f) $1 \times 6 \% 800{ }^{\circ} \mathrm{C} 20 \mathrm{~min}$ 
considerable number of dislocation tangles exist near the grain boundary area after $6 \%$ straining and before annealing (Fig. 13b). From Fig. 13(b)-(e), it can be seen that the densities of the dislocation tangles start to decrease after $9 \mathrm{~min}$. According to Fig. 9, only grain growth would occur, rather than recrystallization, for the as-received and $25 \%$ strained samples, thus the dislocation changes seen are indication of a recovery anneal. A further decrease in dislocation density occurred after $14 \mathrm{~min}$, accompanied by the start of grain growth. The dislocation tangles disappeared after 16-20 min of annealing, corresponding to the middle and the end of grain growth, respectively.

These observations supported the core-mantle mechanism proposed by Thompson et al. [15] and the TEM observations of Murr and Wang [19], who showed a similar micrograph in Ni to Fig. 13(b) with the formation of dislocation cells. At strain levels greater than about $12 \%$, they also showed that there was no observable difference in dislocation density relative to the core or mantle. Thus, a recovery anneal of the $6 \%$ strained $\mathrm{Ni}$ would lead to the annihilation of these extrinsic boundary dislocations and grain boundary migration which is necessary for twin generation following a low pre-strain. As mentioned above, this concept has been modelled by Sangal and Tangri [18] where during an anneal non-equilibrium grain boundaries can be transferred to an equilibrium state via grain boundary migration. This leads to the EGBDs which are stored during deformation, upon annealing being capable of forming special boundaries in the $\Sigma 3-29$ range via grain boundary migration [18].

Relative comparison of the present data of low strain $(6 \%)$ versus medium strain $(25 \%)$ is possible from the literature [5]. In the present research, the single low strain route resulted in special boundary fractions close to $80 \%$, whereas the medium strain processing resulted in a fraction special of $35-40 \%$ at $800{ }^{\circ} \mathrm{C}$, similar to the as-received material. In other words, no benefit was seen in increasing the special process fraction for the single $25 \%$ strain. The data from Randle and Coleman [5] however does not agree with the present results, in that the CSL percentage of $\Sigma 3$, 9, and 27 increased to about 58 from an as-received value of about 28. Possible reasons for this difference are discussed below.

The first difference between the two sets of results is in the materials used, commercially pure $\mathrm{Ni}(99.5 \%)$ in the present communication and $99.99 \% \mathrm{Cu}$ for Randle and Coleman. Randle and Coleman [5] also used tensile straining rather than rolling, coupled with difference in heat treatments relative to their melting points, as well as times of annealing. Another major difference is the stacking fault energy (SFE) of $\mathrm{Cu}$ being about $45 \mathrm{~mJ} / \mathrm{m}^{2}$ versus a best estimate for $\mathrm{Ni}$ of $128 \mathrm{~mJ} / \mathrm{m}^{2}$ [20]. As the SFE is well known to influence the dislocation configurations and densities, the properties of metals and alloys can be greatly influenced by the SFE value. Thus, the effect of strain in a low versus a high SFE material can be quite different, as pointed out by Murr and Wang for 304 stainless steel and $99.98 \% \mathrm{Ni}$ [19]. A final point one should consider is that interactions between processing parameters, such as strain, time, and temperature, rather than considerations of the individual parameters alone, can have a profound effect on the generation of special boundaries in Ni [21], and should be considered in schedule developments to optimize $F_{\mathrm{sp}}$ fractions.

\section{Conclusions}

A comparison has been made using commercially pure $\mathrm{Ni}$ of the effect of processing via a single low strain recovery route to optimize the fraction of special boundaries and a single high strain, fully recrystallized route. The following conclusions were observed:

(1) An optimized low strain processing route of $1 \times 6 \%$ strain, annealed for $30 \mathrm{~min}$ at $800{ }^{\circ} \mathrm{C}$, resulted in a fraction of special boundaries of $78 \%$ compared to the as-received condition of $35 \%$

(2) A high strain processing route of $1 \times 25 \%$ strain, annealed at $800{ }^{\circ} \mathrm{C}$ for $30 \mathrm{~min}$ produced a special fraction of $38 \%$, similar to the as-received value.

(3) The low strain route resulted in a recovery anneal condition, unlike the high strain route which resulted in recrystallization. The low strain and anneal route caused grain boundary migration, leading to the generation of $\Sigma 3^{n}$ special boundary types over and above that shown by the as-received $\mathrm{Ni}$.

(4) The generation of increased special boundary fraction in the low strain material accentuated the breakup of the random boundary network relative to the asreceived and the high strain material.

(5) A reasonable combination of strain, time, and temperature resulting in grain boundary migration that is an efficient way to generate a high number of special boundaries in C.P. Ni.

(6) Differences in the behavior of the low strain $6 \%$ route versus the $25 \%$ route to increase the special boundary and fractions are based on theories related to dislocation generation from grain boundaries.

Acknowledgments The authors would like to acknowledge the financial support for the research from the Natural Sciences and Research Council (NSERC), Canada, in the form of a Discovery Grant to Dr. Richards. The technical support of Mike Boskwick, John van Dorp and Don Mardis is also gratefully acknowledged. 


\section{References}

1. M. Kumar, W. King, A. Schwartz, Modifications to the microstructural topology in FCC materials through thermomechanical processing. Acta Mater. 48, 2081-2091 (2000)

2. S.-L. Lee, N.L. Richards, The effect of single-step low strain and annealing of nickel on grain boundary character. Mater. Sci. Eng. A390, 81-87 (2005)

3. S.-L. Lee, N.L. Richards, Influence of long term annealing on grain boundary character distribution in nickel. Mater. Sci. Eng. A405, 74-85 (2005)

4. M. Shimada, H. Kokawa, Z.J. Wang, Y.S. Sato, I. Karibe, Optimization of grain boundary character distribution for intergranular corrosion resistant 304 stainless steel by twin-induced grain boundary engineering. Acta Mater. 50(9), 2331-2341 (2002)

5. V. Randle, M. Coleman, A study of low-strain and medium strain grain boundary engineering. Acta Mater. 57, 3410-3421 (2009)

6. L. Lim, R. Raj, On the distribution of $\Sigma$ for grain boundaries in polycrystalline nickel prepared by strain-annealing technique. Acta Metall. 32, 1177-1181 (1984)

7. G.S. Was, V. Thaveeprungsriporn, D.C. Crawford, Grain boundary misorientation effects on creep and cracking in Nibased alloys. JOM 50(2), 44-49 (1990)

8. Q. Li, B.M. Guyot, N.L. Richards, Effect of processing parameters on grain boundary modifications to alloy Inconel 718. Mat. Sci. Eng. A 458, 58-66 (2007)

9. R.J. Romero, L.E. Murr, Deformation-induced microstructure and martensite effects on transgranular carbide precipitation in type 304 stainless steels. Acta Metall. Mater. 43, 461-469 (1995)

10. V. Randle, Mechanism of twin-induced grain boundary engineering in low stacking fault energy materials. Acta Mater. 47(15), 4187-4196 (1999)
11. G. Palumbo, United States Patent 5817193, October, 1998

12. D.S. Lee, A grain boundary engineering approach to promote special boundaries in Pb-base alloy. Mat. Sci. and Eng. A354, 106-111 (2003)

13. B. Alexandreanu, G.S. Was, A priori determination of the sampling size for grain-boundary character distribution and grainboundary degradation analysis. Philos. Mag. A81(8), 1951-1965 (2001)

14. B. Guyot, N.L. Richards, A study of the effect of cold rolling and annealing on special grain boundary fractions in commerciallypure nickel. Mater. Sci. Eng. A395, 87-97 (2005)

15. A.W. Thompson, M.I. Baskes, W.F. Flanaghan, The dependence of polycrystal work hardening on grain size. Acta Metall. 21, 1017-1028 (1973)

16. M.A. Meyers, E. Ashworth, A model for the effect of grain size on the yield stress of metals. Phil. Mag. 46(5), 737-759 (1982)

17. M.F. Ashby, Deformation of plastically non-homogeneous materials. Phil. Mag. 21(170), 399-424 (1970)

18. S. Sangal, K. Tangri, Effect of small plastic deformation and annealing on the properties of polycrystals: part 11 . Theoretical model for the transformation of non-equilibrium grain boundaries. Met. Trans. 20(3), 479-484 (1989)

19. L.E. Murr, S.-H. Wang, Comparison of microstructural evolution associated with the stress-strain diagrams for nickel and 304 stainless steel: an electron microscopy study of microyielding and plastic flow. Res. Mech. 4, 237-274 (1992)

20. Q. Li, J.R. Cahoon, N.L. Richards, On the calculation of annealing twin density. Scripta Mater. 55, 1155-1158 (2006)

21. Q. Li, N.L. Richards, Quantitative effects of strain, temperature, number of cycles and grain size, on the fraction of special boundaries generated in commercially pure nickel. Can. Met. Q. 51-52, 195-201 (2012) 